\title{
Engaging Aldehydes in CuH-Catalyzed Reductive Coupling Reac- tions: Stereoselective Allylation from 1,3-Diene Pronucleophiles
}

\author{
Chengxi Li, ${ }^{\dagger}$ Kwangmin Shin,${ }^{\dagger}$ Richard Y. Liu, and Stephen L. Buchwald* \\ Department of Chemistry, Massachusetts Institute of Technology, Cambridge, Massachusetts 02139, United States
}

\begin{abstract}
Recently, CuH-catalyzed reductive coupling processes involving carbonyl compounds and imines has become an attractive alternative to traditional methods for stereoselective addition to carbonyls due to the ability to use readily accessible and stable olefin-derived pronucleophiles as surrogates for organometallic reagents. However, the inability to use aldehydes, which traditionally reduce too rapidly in the presence of copper hydride complexes to be viable substrates, has been a major limitation. We show that by exploiting relative concentration effects through slow addition, we can invert this intrinsic reactivity and achieve the reductive coupling of 1,3-dienes with aldehydes. Using this method, both aromatic and aliphatic aldehydes can be transformed to valuable products with high levels of diastereo- and enantioselectivity and in the presence of many useful functional groups. Furthermore, using a combination of theoretical (DFT) and experimental methods, important mechanistic features of this reaction related to stereo- and chemoselectivity were uncovered.
\end{abstract}

\section{INTRODUCTION}

The addition of nucleophilic organometallic reagents, such as those based on $\mathrm{Mg}$ (Grignard), B, Si, Sn and $\mathrm{Zn}$, to carbonyl derivatives is a key reaction for $\mathrm{C}-\mathrm{C}$ bond formation. ${ }^{1}$ Accordingly, the development of methods to accomplish this transformation in a catalytic, stereoselective manner have been the subject of widespread research efforts. ${ }^{2}$ Recently, breakthroughs in transition metal catalysis have enabled the use of olefin-derived nucleophiles, one of the most convenient and readily available classes of compounds, as surrogates of traditional organometallic reagents in carbonyl addition processes ${ }^{3-10}$ Following pioneering work by Krische using $\mathrm{Rh}^{4 \mathrm{a}, \mathrm{b}}, \mathrm{Ru}^{5}, \mathrm{Ir}^{6}$, and $\mathrm{Ni}$ catalysts, ${ }^{7 \mathrm{e}}$ several research groups, including ours, have developed a number of $\mathrm{CuH}$-catalyzed processes for reductive $\mathrm{C}-\mathrm{C}$ bond formation from $\pi$-unsaturated pronucleophiles (Figure 1A). ${ }^{9,10}$

Each of these metals is associated with distinct catalytic mechanisms and complementary reactivity, and hence, features respective advantages and deficiencies. In the case of $\mathrm{Cu}$, the most notable advantages include mild conditions, high stereoselectivity, and exceptional tolerance for polar functional groups. ${ }^{9,10}$ On the other hand, the proclivity of $\mathrm{CuH}$ intermediates to participate in direct reduction of carbonyl compounds has so far limited the generality of this strategy: to date, only the functionalization of ketones and imines using relatively activated alkenes such as allenes, enynes, styrenes have been successfully accomplished (Figure 1B). ${ }^{9,10}$ The conspicuous absence of aldehydes, the most common class of electrophiles in carbonyl addition reactions, can be explained by the rate of their direct reaction with $\mathrm{CuH}$ species, which is sufficiently high that the olefinic partner typically does not have the chance to participate in hydrocupration. ${ }^{11}$

With the aim of addressing this important limitation, we herein report the expansion of the scope of $\mathrm{CuH}$-catalyzed reductive olefin-carbonyl coupling to aldehyde starting materials, using a combination of ligand-conferred regioselectivity and kinetic control through metered addition. To prove our concept, we selected 2-substituted 1,3-dienes as relatively unactivated model pronucleophiles (Figure 1B). 2-Substituted allyl groups are difficult to introduce using stoichiometric organometallic reagents, and few catalytic methods have been reported for their stereoselective installation. ${ }^{2,12}$

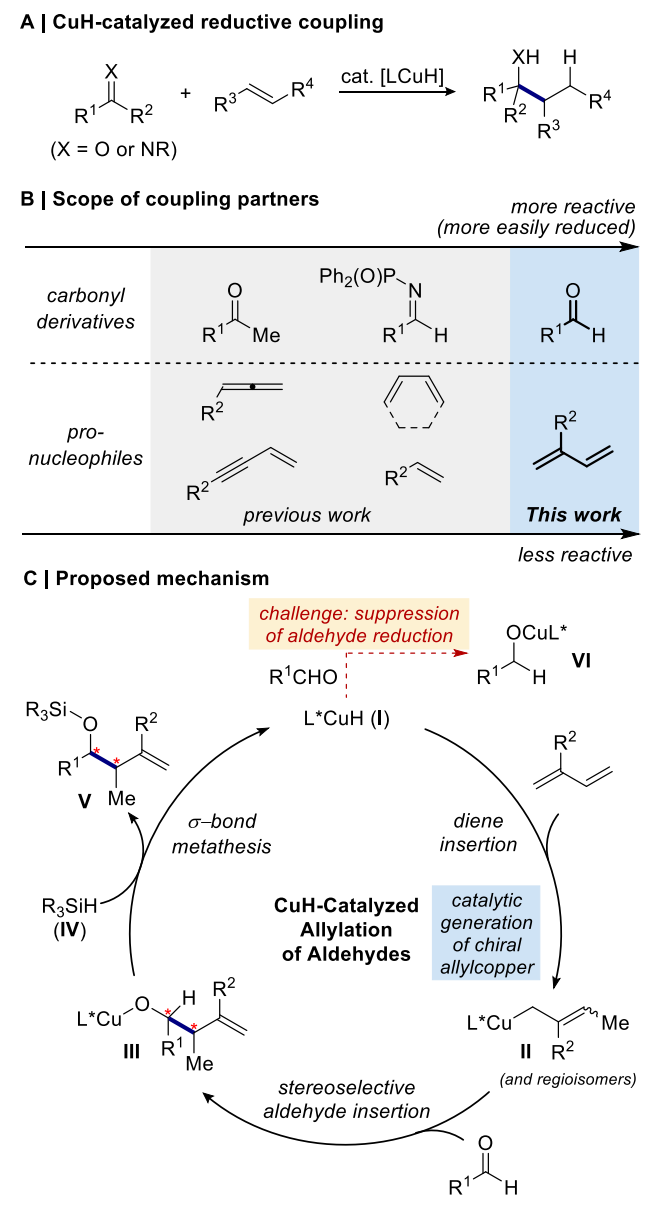

Figure 1. Overview of $\mathrm{CuH}$-catalyzed reductive coupling of $\pi$ unsaturated pronucleophiles with carbonyl derivatives. 
Considering previous mechanistic studies, ${ }^{9 f}$ we envisioned that our proposed transformation would proceed through hydrocupration of a diene to generate a mixture of allylcopper complexes represented by II (Figure 1C). One or more of these species could engage an aldehyde coupling partner in a stereoselective migratory insertion process to form the copper alkoxide III, from which metathesis with a hydrosilane (IV) would regenerate $\mathrm{LCuH}(\mathbf{I})$ and the desired product $(\mathbf{V})$ in silyl-protected form. Clearly, the diene hydrocupration must be faster than the rapid direct reduction of the aldehyde, which is contrary to the intrinsic kinetic preferences of these elementary reactions $(18.5$ vs. $13.9 \mathrm{kcal} / \mathrm{mol}$ calculated free energy barriers for diene and aldehyde hydrocupration respectively, see below). In this article, we describe the optimization of a reaction system that displays this inverted chemoselectivity, its applications to the highly regio-, diastereo- and enantioselective allylation of both aliphatic and aromatic aldehydes, and mechanistic studies that explain the origin of these selectivities.

\section{- RESULTS AND DISCUSSION}

We initiated our study by examining reactions of $p$ anisaldehyde (1a) and 2-phenyl-1,3-butadiene (1b) under reaction conditions previously described for $\mathrm{CuH}$-catalyzed ketone allylation. ${ }^{9 \mathrm{e}, \mathrm{f}}$ With $(S, S)$-QuinoxP*(L1) as the ligand, the desired product 1 was obtained in $48 \%$ yield, with exclusive branched-selectivity and excellent preference for the indicated diastereomer (11:1 dr). However, the major diastereomer was formed with only a moderate level of enantioselectivity (68:32 er, Table 1, entry 1). No desired homoallylic alcohol 1 was obtained when $(R)$-DTBM-SEGPHOS (L2) was used as the ligand: complete reduction of the aldehyde was observed instead (Table 1, entry 2). JosiPhos ${ }^{13}$ derivative SL-J011-1 (L3), a ligand which had been employed with good results in ketone allylation, ${ }^{9 \mathrm{f}}$ was also examined. The corresponding test reaction provided 1 in moderate $(57 \%)$ yield, with 8:1 dr and 85:15 er (Table 1, entry 3). Further evaluation of commercially available common chiral ligands revealed $(S, S)$-Ph-BPE $(\mathbf{L 4})$ to be optimal (Table 1, entry 4$)$, providing $75 \%$ yield of 1 was obtained with excellent $\mathrm{dr}$ (21:1) and er (96.5:3.5).

Table 1. Evaluation of Reaction Conditions for the $\mathrm{CuH}$-Catalyzed Allylation of 4-Methoxybenzaldehyde. ${ }^{a}$

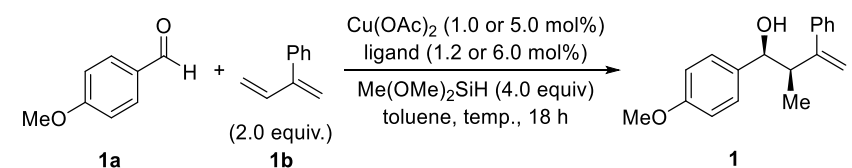

\begin{tabular}{|c|c|c|c|c|c|c|}
\hline Entry & Ligand & $\begin{array}{l}\text { Cat. } \\
(\%)\end{array}$ & $\begin{array}{c}\text { Temp. } \\
\left({ }^{\circ} \mathrm{C}\right)\end{array}$ & $\begin{array}{l}\text { Yield }^{b} \\
1(\%)\end{array}$ & dr & $\begin{array}{c}\text { er }^{c} \\
\text { (major) }\end{array}$ \\
\hline 1 & L1 & 5 & $\mathrm{rt}$ & 48 & $11: 1$ & $68: 32$ \\
\hline 2 & L2 & 5 & $\mathrm{rt}$ & 0 & -- & -- \\
\hline 3 & L3 & 5 & $\mathrm{rt}$ & 57 & $8: 1$ & $85: 15$ \\
\hline 4 & L4 & 5 & $\mathrm{rt}$ & 75 & $21: 1$ & $96.5: 3.5$ \\
\hline 5 & L4 & 5 & 40 & 71 & $24: 1$ & $95.5: 4.5$ \\
\hline 6 & L4 & 1 & $\mathrm{rt}$ & 90 & $13: 1$ & $94: 6$ \\
\hline 7 & L4 & 1 & 0 & 94 & $13: 1$ & $95: 5$ \\
\hline $8^{d}$ & L4 & 1 & 0 & 33 & $9: 1$ & $95: 5$ \\
\hline $9^{e}$ & L4 & 1 & 0 & 97 & $10: 1$ & 91:9 \\
\hline
\end{tabular}


and enantioselectivity (>20:1 dr, 99.5:0.5 er). Finally, a naturally occurring diene, myrcene, proved to be an effective reagent, providing $\mathbf{2 0}$ with good yield and useful stereoselectivity.

Table 2. Evaluation of the Scope of the Aldehyde Allylation with Branched Dienes. ${ }^{a}$

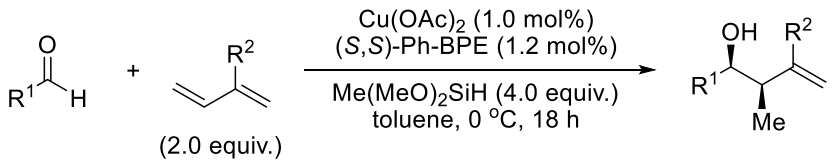

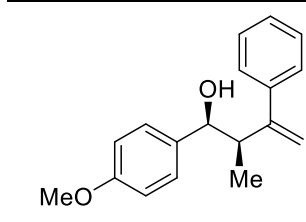

1<smiles>C=C(c1ccccc1)[C@H](C)[C@H](O)c1ccccc1</smiles>

$87 \%$ yield, $11: 1 \mathrm{dr}, 95.5: 4.5 \mathrm{er}$<smiles>C=C(c1ccccc1)[C@H](C)[C@H](O)C1CCCCC1</smiles>

5

$82 \%$ yield, $>20: 1 \mathrm{dr}, 97: 3 \mathrm{er}$<smiles>C=C(c1cccc(F)c1)[C@H](C)[C@H](O)c1ccsc1</smiles>

9

$85 \%$ yield, $7: 1 \mathrm{dr}, 97: 3 \mathrm{er}$<smiles>C=C(c1ccsc1)[C@H](C)[C@H](O)C1CCCCC1</smiles>

13

$81 \%$ yield, $>20: 1 \mathrm{dr}, 96: 4$ er<smiles>C=C(C1CCCCC1)[C@@H](C)[C@H](O)C1CC1</smiles>

17<smiles>C=C(c1ccccc1)[C@H](C)[C@H](O)C(C)(C)C</smiles>

6

$87 \%$ yield, $>20: 1 \mathrm{dr}, 99.5: 0.5 \mathrm{er}$<smiles>C=C(c1cccc(F)c1)[C@H](C)[C@@H](O)c1cn(C(=O)OCCC)c2ccccc12</smiles>

10

$79 \%$ yield, 8:1 dr, 99:1 er<smiles>C=C(c1cn(C(=O)OCC)c2ccccc12)[C@H](C)[C@@H](C)O</smiles>

14<smiles>C=C(c1ccccc1)[C@H](C)[C@H](O)CCc1ccccc1</smiles>

3

$68 \%, 9: 1 \mathrm{dr}, 98: 2 \mathrm{er}$

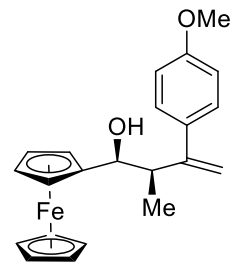

7

$90 \%$ yield, $7: 1 \mathrm{dr}, 95: 5 \mathrm{er}$<smiles>C=C(c1ccsc1)[C@H](C)[C@H](O)c1ccc(S(C)(=O)=O)cc1</smiles>

11

$92 \%$ yield, $6: 1 \mathrm{dr}, 94: 6$ er<smiles>C=C(C1CCCCC1)[C@@H](C)[C@@H](O)/C(=C/c1ccccc1)CCCCC</smiles>

15

$81 \%$ yield, $10: 1 \mathrm{dr}, 95: 5 \mathrm{er}$<smiles>C=C([C@@H](C)[C@@H](O)C(C)(C)C)C12CC3CC(CC(C3)C1)C2</smiles>

19<smiles>C=C(c1ccccc1)[C@H](C)[C@@H](O)C[C@@H](C)CCC=C(C)C</smiles>

4

$77 \%$ yield, $>20: 1 \mathrm{dr}$<smiles>C=C(c1ccc(OC)cc1)[C@H](C)[C@H](O)c1ccoc1</smiles>

8

$86 \%$ yield, $8: 1 \mathrm{dr}, 97: 3$ er<smiles>C=C(c1ccsc1)[C@@H](C)[C@H](C)O</smiles>

12

$84 \%$ yield, $>20: 1 \mathrm{dr}, 99: 1 \mathrm{er}$<smiles>C=C(C1CCCCC1)[C@@H](C)[C@H](C)O</smiles>

16

$50 \%$ yield, $>20: 1 \mathrm{dr}, 93.5: 6.5$ er

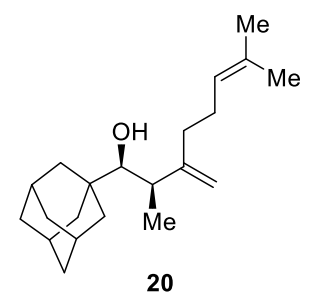

$73 \%$ yield, 5:1 dr, 85:15 er

$66 \%$ yield, $14: 1 \mathrm{dr}, 97: 3 \mathrm{er}$

$57 \%$ yield, $>20: 1 \mathrm{dr}, 95: 5$ er

$48 \%$ yield, $>20: 1 \mathrm{dr}, 99.5: 0.5 \mathrm{er}$

${ }^{a}$ Yields indicate the isolated yield of product as a mixture of two diastereomers on a $1.0 \mathrm{mmol}$ scale. Diastereomeric ratios were determined by ${ }^{1} \mathrm{H}$ NMR spectroscopy for both the crude and purified products; enantiomeric ratios were determined by HPLC, SFC or chiral GC analysis on commercial chiral columns. Yields, diastereomeric ratios, and enantiomeric ratios are the averages for two identical runs. See Supporting Information for full details. 


\section{MECHANISTIC STUDIES}

By analogy to other copper-catalyzed reductive olefin-keton $^{9 \mathrm{~b}, \mathrm{f}}$ and olefin-imine coupling ${ }^{\mathrm{aa}}$ reactions, we proposed that the current reaction proceeds through the mechanism illustrated in Figure 1C. Previous computational and experimental investigations of related transformations have revealed, in intricate detail, the sequence of events and controlling factors that dictate the regio-, diastereo- and enantioselectivity observed in these transformations. ${ }^{9 f}$ However, we were interested in several questions pertaining to the mechanism and selectivity that are particular to this aldehyde allylation. First, we wanted to confirm the feasibility of our proposed series of elementary steps, as well as their relative rates, their reversibility, and the identity of the selectivity-determining step(s). It is important to note, however, that metered addition of the aldehyde, which presumably maintains a low steady-state concentration of aldehyde, is crucial to obtaining high yields of product. Thus, it is necessary that our model to account for this effect. Second, we hoped to explain the stereochemical outcome of our reaction. Specifically, our rationale should both identify the step(s) that control the diastereo- and enantioselectivity (allylcopper isomerization, $\mathrm{C}-\mathrm{C}$ bond formation, $\sigma$-bond metathesis, or a combination thereof), as well as the specific ligand-substrate interactions responsible for destabilizing the disfavored stereoisomeric pathways. Finally, we wanted to confirm experimentally that competing aldehyde vs. diene insertion into a copper(I) hydride complex determines the chemoselectivity with regard to reduction vs. the desired coupling.

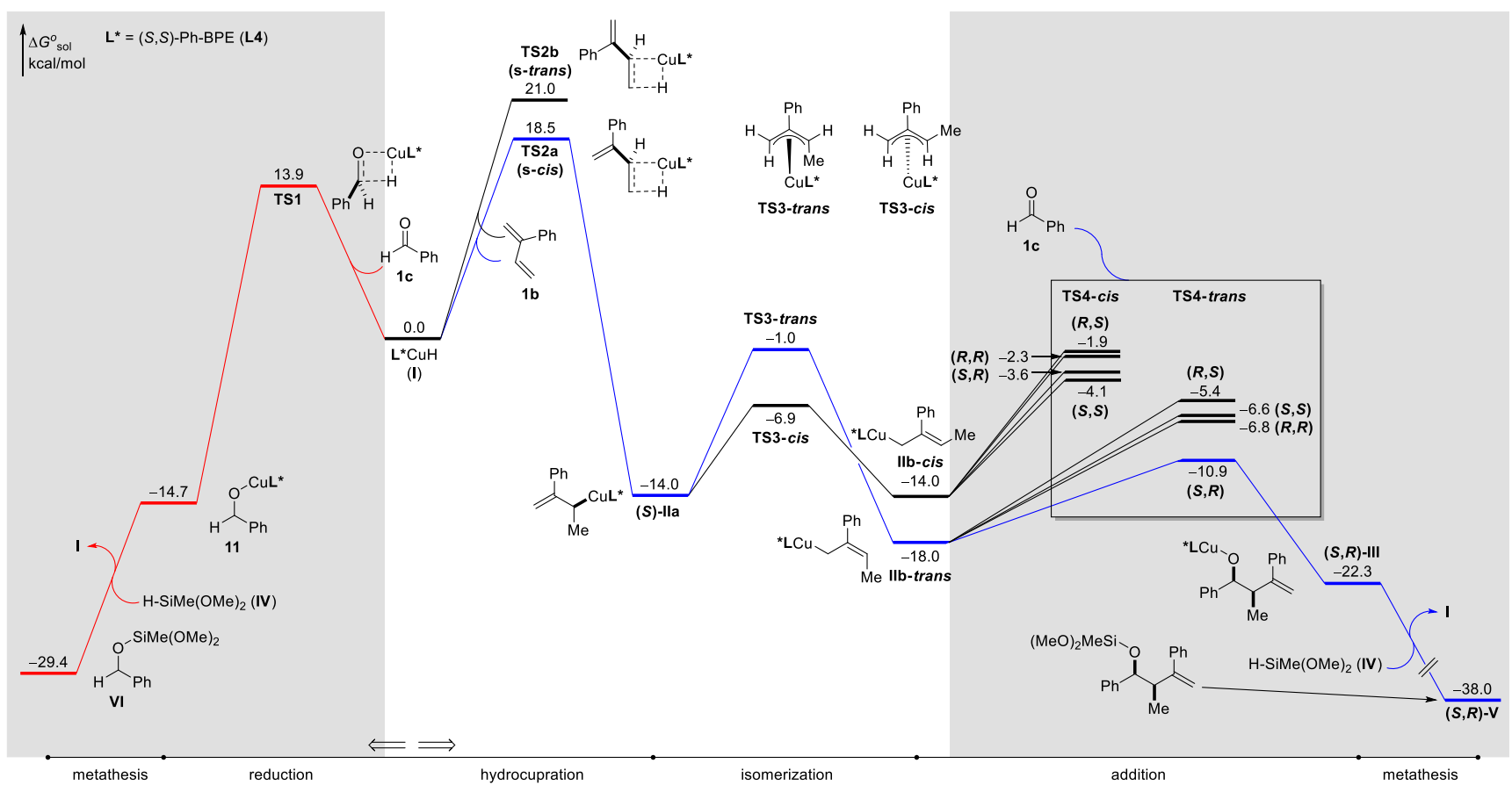

Figure 2. Computed energy profiles for $\mathrm{CuH}$-catalyzed allylation (blue) and reduction (red) of benzaldehyde (1c). These calculations were performed at the M06-2X/SDD-6-311+G(d,p)/SMD(toluene)//B3LYP/SDD-6-31G(d) level of theory. Standard free energies are relative to infinitely separated $\mathbf{I}$ and reactants (1c and 1b). See the Supporting Information for details.

\section{$(S, R)$-TS4-trans favored}

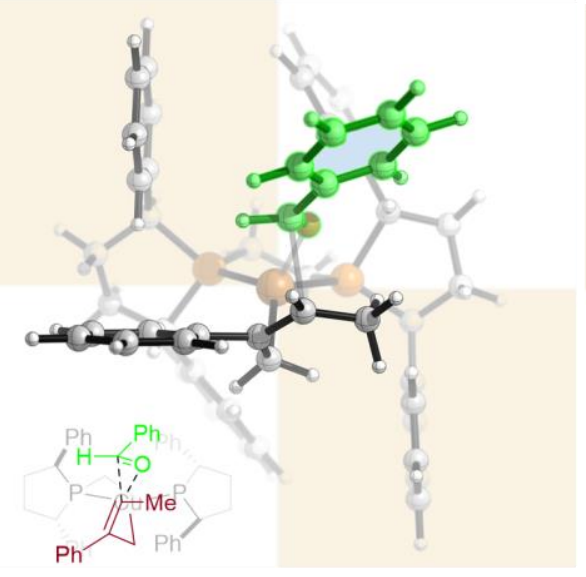

$(R, S)-T S 4-t r a n s$

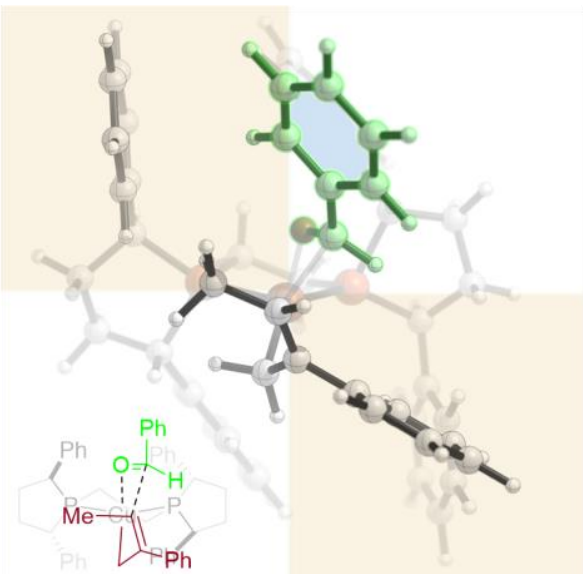

$(R, R)$-TS4-trans

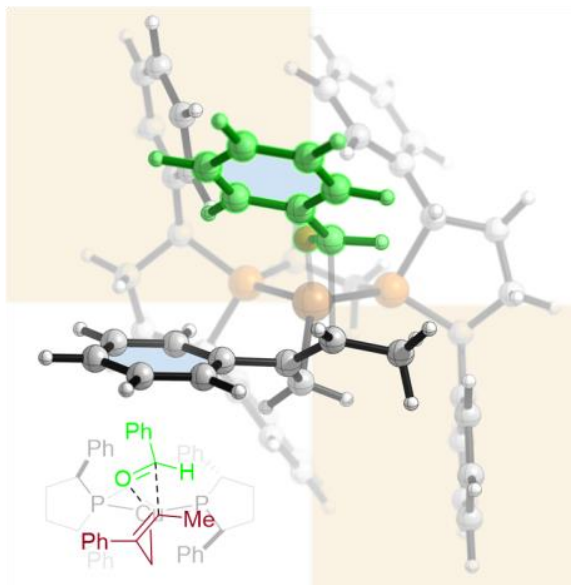


Figure 3. Stereochemical model for the $\mathrm{CuH}$-catalyzed aldehyde allylation process. DFT-optimized lowest energy transition state structures for the $\mathrm{C}-\mathrm{C}$ bond formation step, leading to the major stereoisomer (left), the minor diastereomer (middle) and the minor enantiomer (right) of product 2 . See the Supporting Information for details.

We first turned to DFT-based modeling to address the first two questions. The computed Gibbs free energy profile, using model diene $\mathbf{1 b}$ and benzaldehyde (1c) as model substrates, is shown in Fig. 2. Starting from $\mathbf{L} * \mathrm{CuH}(\mathbf{I})$, where $\mathbf{L}^{*}=(S, S)$ $\mathrm{Ph}$-BPE, irreversible hydrocupration of $\mathbf{1 b}$ takes place preferentially through its s-cis conformation and with facial selectivity to generate the $S$ enantiomer of branched allylcopper complex IIa. We found that $(S)$-IIa can isomerize rapidly to a nearly isoenergetic linear isomer IIb-cis, in which the $\mathrm{Ph}$ and $\mathrm{Me}$ alkene substituents are mutually cis. While this isomerization is associated with an energetic barrier of $7.1 \mathrm{kcal} / \mathrm{mol}$ (TS3-cis), there also exists a second, slower isomerization pathway (13.0 $\mathrm{kcal} / \mathrm{mol}$ barrier) leading to more stable linear isomer IIb-trans (4.0 kcal/mol more stable than IIa), in which the $\mathrm{Ph}$ and Me are mutually trans.

In accordance with the Curtin-Hammett principle, the isomer of II (e.g., IIa, IIb-cis, IIb-trans) through which the reaction proceeds will depend on the rate of the subsequent addition step relative to the isomerization processes. However, prior to further elaboration on this aspect, we first need to more precisely consider the effect of slow addition of aldehyde on the mechanistic model in general. While it is conventional to plot energy diagrams using standard thermodynamic parameters, (i.e., those at $1 \mathrm{M}$ solution for solutes), true relative free energies depend on the concentrations of the species involved. In cases where two reactants might have concentrations differing by many orders of magnitude during steady-state catalyst turnover, it is particularly important to consider relative concentration effects in the interpretation of an energy diagram. ${ }^{15}$

Accordingly, in the reaction under consideration, we noted that the metered addition of aldehyde over several hours should ensure that the concentration of the aldehyde is very low relative to the other reactants. The presence of this concentration disparity is equivalent to raising the free energy of all states wherein aldehyde is associated with the catalyst (Figure 2, highlighted in grey) relative to states in which the aldehyde is free (in white). Indeed, we find that if, by relative concentration effects, TS1 is raised in energy by more than about $4.6 \mathrm{kcal} / \mathrm{mol}$ relative to TS2a, hydrocupration can be favored over direct reduction of the aldehyde, as is observed in the reaction.

A less obvious consequence is that the reaction of the copper allyl species is also slowed, which has implications on reversibility of allylcopper isomerization. We examined eight diastereomeric transition states for allylcopper addition to the aldehyde (Figure 4, boxed and labeled TS4-cis/trans). Considering standard free energies alone, it would appear that reaction of the cis-allyl complex IIb-cis through $(\boldsymbol{S}, \boldsymbol{S})$-TS4-cis is sufficiently facile that competing isomerization to the more stable IIb-trans should be kinetically precluded. However, with species highlighted in grey raised in energy by the amount $(>4.6$ $\mathrm{kcal} / \mathrm{mol}$ ) required to avoid direct aldehyde reduction, reaction of IIb-cis with aldehyde through the TS4-cis transition states necessarily become more challenging than reversible isomerization to IIb-trans. In this scenario, the predicted major reaction pathway (highlighted in blue) proceeds through $(S, R)$-TS4trans, leading to the diastereomer of product that is experimentally observed to be predominant $((S, R)-\mathbf{V})$.
We next examined the transition state structures to elucidate the origin of stereoselectivity in the addition step. Monomeric complexes of Ph-BPE-ligated copper are known adopt a conformation whose steric profile is well approximated by a quadrant model. ${ }^{16}$ For instance, in the preferred six-membered cyclic transition state (Figure 3, left panel) ${ }^{17}$ the largest substituents of both the allyl component and the aldehyde component are directed into the less hindered (white) quadrants during the $\mathrm{C}-\mathrm{C}$ bond formation. In contrast, minor enantiomer of product can form if the aldehyde attacks the opposite face of the diene (Figure 3, center panel). However, a steric clash is created between the aryl substituent of the diene and the Ph substituent of the ligand (bottom-right quadrant), which destabilizes this structure. Furthermore, unfavorable steric interactions between the aldehyde substituent and the other $\mathrm{Ph}$ group on the ligand (top-left quadrant) causes the structure to distort from its ideal, chair-shaped cyclic geometry. Finally, a minor diastereomer can form if, relative to the favored transition state, the opposite face of the aldehyde is attacked (Figure 3, right panel). The dominant destabilizing interaction in this case is between the aldehyde substituent and the $\mathrm{Ph}$ group of $\mathrm{Ph}-\mathrm{BPE}$ in the top-left quadrant. Overall, this model determines the correct sense of selectivity, although the magnitude is somewhat overestimated relative to experiment (95:5 er, 5:1 dr).

We also performed kinetic experiments to further explore the effect of slow addition on the chemoselectivity of our reaction (Figure 4). Under the standard conditions, except with no diene present, the reduction of aldehydes is extremely rapid. In our experiment, benzaldehyde was fully consumed within 40 min, forming the silyl-protected benzyl alcohol (Figure 4, top panel). If a single equivalent of diene is added at the beginning of the reaction, the corresponding reductive coupling product is observed to form in a roughly 1:9 ratio with the reduction product (Figure 4, middle panel). Notably, however, the overall consumption of starting material has been retarded, with the reaction now requiring over $3 \mathrm{~h}$ to reach full consumption of starting material. Finally, when a large excess of diene is added at the beginning of the reaction (5.0 equiv diene, Figure 4, bottom panel), the reductive coupling product is observed to form at a higher ratio relative to the reduction product. Simultaneously, the total consumption of starting material is further retarded.

Two conclusions can be drawn from these data. First, the correlation of the product-to-reduction ratio with the diene-toaldehyde concentration ratio supports the proposed the role of slow addition in our mechanistic scheme. In Figure 2, higher concentration of diene lowers the energy of the blue pathway relative to the red pathway, and therefore leads to increased formation of the coupling product. Second, the seemingly inhibitory effect of the diene explains why the slow addition protocol must be conducted over a relatively long timespan $(\sim 3.5 \mathrm{~h})$, in spite of the fact that we are trying to outcompete an extremely rapid reaction (half-life on the order of several min). Under the conditions of our protocol, at large excesses of diene relative to aldehyde, the rate of both the reduction and desired coupling are slow. 


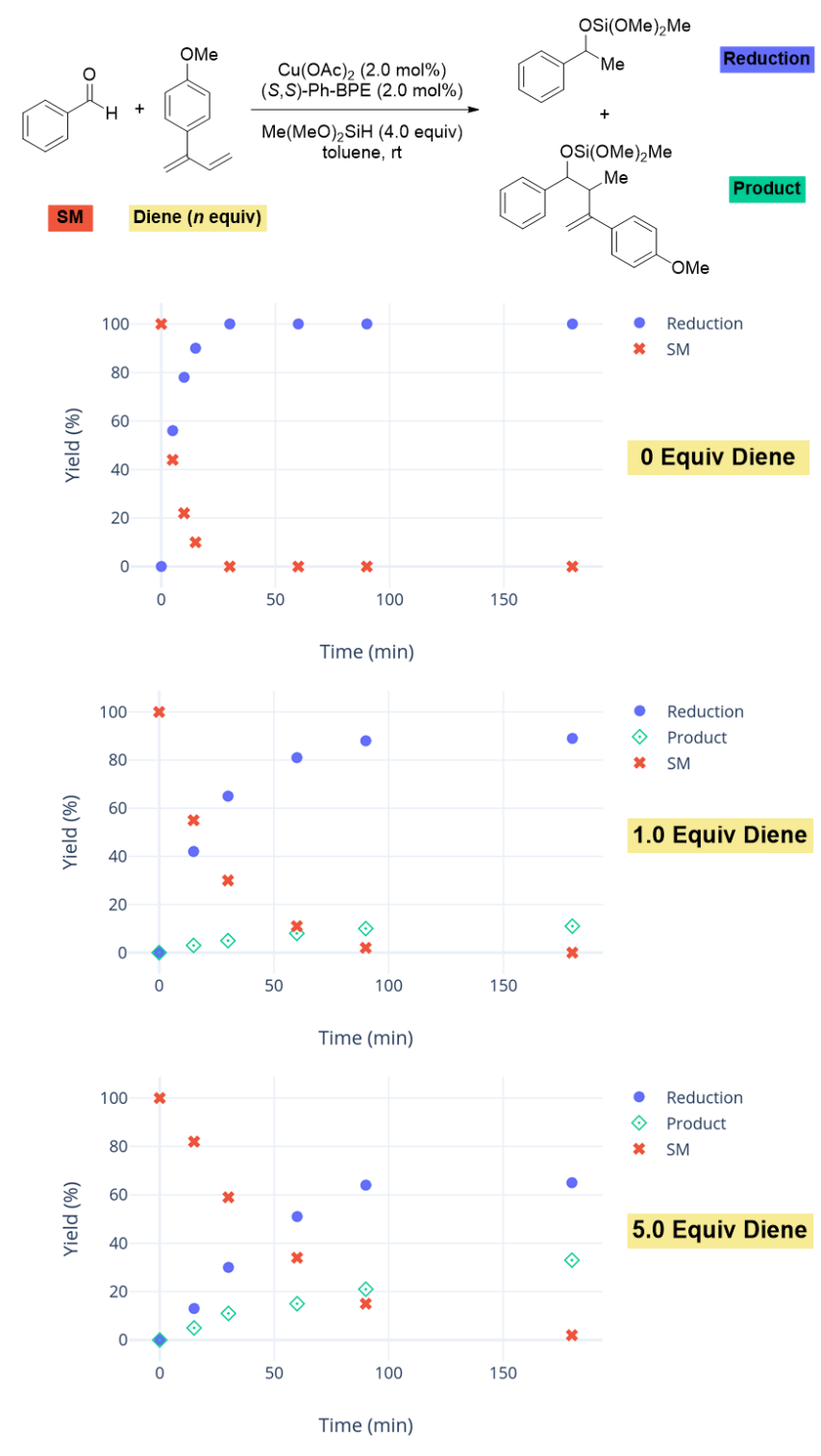

Figure 4. Kinetic profiles of the competing reductive coupling and reduction processes as a function of diene concentration. See the Supporting Information for experimental details.

\section{- CONCLUSION}

In summary, we have developed a highly efficient coppercatalyzed allylation of aldehydes using dienes as allylmetal surrogates. Computational studies were performed which indicate that a reversible isomerization of copper(I) allyl species is formed, from which reaction of the trans-linear isomer with the aldehyde yields the major stereoisomer of product. Transition state models are provided, which show the specific steric interactions between ligand and substrates that are responsible for the stereoselectivity. Finally, kinetic experiments were performed, demonstrating effect of aldehyde-diene relative concentration on the chemoselectivity of this reaction.

\section{ASSOCIATED CONTENT}

The Supporting Information is available free of charge on the ACS Publications website at DOI: 10.1021 jacs.
Experimental procedures and characterization data for all compounds (PDF)

NMR spectra (PDF)

SFC and HPLC traces (PDF)

Computational details and Cartesian coordinates of optimized geometries (PDF)

\section{AUTHOR INFORMATION}

\section{Corresponding Author}

*sbuchwal@mit.edu

\section{ORCID}

Chengxi Li: 0000-0003-3904-0299

Kwangmin Shin: 0000-0002-1708-2351

Richard Y. Liu: 0000-0003-0951-6487

Stephen L. Buchwald: 0000-0003-3875-4775

\section{Author Contribution}

$\dagger$ These authors contributed equally.

\section{Notes}

The authors declare no competing financial interest.

\section{ACKNOWLEDGMENT}

Research reported in this publication was supported by the National Institutes of Health (GM122483, GM058160-17S1) and Basic Science Research Program through the National Research Foundation of Korea (NRF) funded by the Ministry of Education (2018R1A6A3A03011441, K.S.). The content of this communication solely reflects the research and opinion of the authors and does not necessarily represent the official views of the NIH. Solvias AG is acknowledged for a generous gift of SLJ011-1 and Nippon Chemical is thanked for a kind donation of $(S, S)$-QuinoxP*. We are grateful to Dr. Christine Nguyen for advice on the preparation of this manuscript.

\section{REFERENCES}

(1) Schlosser, M. Organometallics in Synthesis: A Manual, $2^{\text {nd }}$ ed.; Wiley: Chichester, UK, 2001.

(2) For selected reviews on addition of organometallic reagents to carbonyl derivatives, see: (a) Pu, L.; Yu, H.-B. Catalytic Asymmetric Organozinc Additions to Carbonyl Compounds. Chem. Rev. 2001, 101, 757-824. (b) Denmark, S. E.; Fu, J. Catalytic Enantioselective Addition of Allylic Organometallic Reagents to Aldehydes and Ketones. Chem. Rev. 2003, 103, 2763-2794. (c) Riant, O.; Hannedouche, J. Asymmetric Catalysis for the Construction of Quaternary Carbon Centres: Nucleophilic Addition on Ketones and Ketimines. Org. Biomol. Chem. 2007, 5, 873-888. (d) Shibasaki, M.; Kanai, M. Asymmetric Synthesis of Tertiary Alcohols and $\alpha$-Tertiary Amines via $\mathrm{Cu}$-Catalyzed $\mathrm{C}-\mathrm{C}$ Bond Formation to Ketones and Ketimines. Chem. Rev. 2008, 108, 2853-2873. (e) Yus, M.; González-Gómez, J. C.; Foubelo, F. Catalytic Enantioselective Allylation of Carbonyl Compounds and Imines. Chem. Rev. 2011, 111, 7774-7854. (f) Yus, M.; González-Gómez, J. C.; Foubelo, F. Diastereoselective Allylation of Carbonyl Compounds and Imines: Application to the Synthesis of Natural Products. Chem. Rev. 2013, 113, 55955698. (g) Beletskaya, I. P.; Nájera, C.; Yus, M. Stereodivergent Catalysis. Chem. Rev. 2018, 118, 5080-5200. 
(3) For selected reviews on reductive coupling of $\pi$-unsaturated pronucleophiles with carbonyl derivatives, see: (a) Nguyen, K. D.; Park, B. Y.; Luong, T.; Sato, H.; Garza, V. J.; Krische, M. J. Metal-Catalyzed Reductive Coupling of Olefin-Derived Nucleophiles: Reinventing Carbonyl Addition. Science 2016, 354, aah5133. (b) Holmes, M.; Schwartz, L. A.; Krische, M. J. Intermolecular Metal-Catalyzed Reductive Coupling of Dienes, Allenes, and Enynes with Carbonyl Compounds and Imines. Chem. Rev. 2018, 118, 6026-6052.

(4) For selected examples of Rh-catalyzed reductive coupling of olefin-derived pronucleophiles with carbonyl compounds, see: (a) Jang, H.; Huddleston, R. R.; Krische, M. J. A New Catalytic $\mathrm{C}-\mathrm{C}$ Bond-Forming Hydrogenation: Reductive Coupling of Dienes and Glyoxals under Catalytic Hydrogenation Conditions. Angew. Chem., Int. Ed. 2003, 42, 4074-4077. (b) Komanduri, V.; Krische, M. J. Enantioselective Reductive Coupling of 1,3-Enynes to Heterocyclic Aromatic Aldehydes and Ketones via Rhodium-Catalyzed Asymmetric Hydrogenation: Mechanistic Insight into the Role of Brønsted Acid Additives. J. Am. Chem. Soc. 2006, 128, 16448-16449. (c) Kimura, M.; Nojiri, D.; Fukushima, M.; Oi, S.; Sonoda, Y.; Inoue, Y. Rh-Catalyzed Reductive Coupling Reaction of Aldehydes with Conjugated Dienes Promoted by Triethylborane. Org. Lett. 2009, 11, 3794-3797. (d) Cooze, C.; Dada, R.; Lundgren, R. J. Formic Acid Mediated Direct ZSelective Reductive Coupling of Dienes and Aldehydes. Angew. Chem., Int. Ed. 2019, Accepted Article.

(5) For selected examples of Ru-catalyzed reductive coupling of $\pi$-unsaturated pronucleophiles with carbonyl compounds, see: (a) Ngai, M.-Y.; Skucas, E.; Krische, M. J. Ruthenium Catalyzed $\mathrm{C}-\mathrm{C}$ Bond Formation via Transfer Hydrogenation: Branch-Selective Reductive Coupling of Allenes to Paraformaldehyde and Higher Aldehydes. Org. Lett. 2008, 10, 2705-2708. (b) Shibahara, F.; Bower, J. F.; Krische, M. J. Ruthenium-Catalyzed $\mathrm{C}-\mathrm{C}$ Bond Forming Transfer Hydrogenation: Carbonyl Allylation from the Alcohol or Aldehyde Oxidation Level Employing Acyclic 1,3-Dienes as Surrogates to Preformed Allyl Metal Reagents. J. Am. Chem. Soc. 2008, 130, 6338-6339. (c) Patman, R. L.; Williams, V. M.; Bower, J. F.; Krische, M. J. Carbonyl Propargylation from the Alcohol or Aldehyde Oxidation Level Employing 1,3-Enynes as Surrogates to Preformed Allenylmetal Reagents: A Ruthenium-Catalyzed C-C Bond-Forming Transfer Hydrogenation. Angew. Chem., Int. Ed. 2008, 47, 5220-5223. (d) Smejkal, T.; Han, H.; Breit, B.; Krische, M. J. All-Carbon Quaternary Centers via Ruthenium-Catalyzed Hydroxy-methylation of 2Substituted Butadienes Mediated by Formaldehyde: Beyond Hydroformylation. J. Am. Chem. Soc. 2009, 131, 10366-10367. (e) Zbieg, J. R.; Yamaguchi, E.; McInturff, E. L.; Krische, M. J. Enantioselective $\mathrm{C}-\mathrm{H}$ Crotylation of Primary Alcohols via Hydrohydroxyalkylation of Butadiene. Science 2012, 336, 324-327. (f) Zbieg, J. R.; Moran, J.; Krische, M. J. Diastereoand Enantioselective Ruthenium-Catalyzed Hydrohydroxyalkylation of 2-Silyl-Butadienes: Carbonyl Syn-Crotylation from the Alcohol Oxidation Level. J. Am. Chem. Soc. 2011, 133, 10582-10586. (g) Park, B. Y.; Montgomery, T. P.; Garza, V. J.; Krische, M. J. Ruthenium Catalyzed Hydrohydroxyalkylation of Isoprene with Heteroaromatic Secondary Alcohols: Isolation and Reversible Formation of the Putative Metallacycle Intermediate. J. Am. Chem. Soc. 2013, 135, 16320-16323. (h) Nguyen, K. D.; Herkommer, D.; Krische, M. J. RutheniumBINAP Catalyzed Alcohol C-H Tert-Prenylation via 1,3Enyne Transfer Hydrogenation: Beyond Stoichiometric
Carbanions in Enantioselective Carbonyl Propargylation. J. Am. Chem. Soc. 2016, 138, 5238-5241.

(6) For selected examples of Ir-catalyzed reductive coupling of $\pi$-unsaturated pronucleophiles with carbonyl compounds, see: (a) Bower, J. F.; Patman, R. L.; Krische, M. J. IridiumCatalyzed $\mathrm{C}-\mathrm{C}$ Coupling via Transfer Hydrogenation: Carbonyl Addition from the Alcohol or Aldehyde Oxidation Level Employing 1,3-Cyclohexadiene. Org. Lett. 2008, 10, 1033-1035. (b) Han, S. B.; Kim, I. S.; Han, H.; Krische, M. J. Enantioselective Carbonyl Reverse Prenylation from the Alcohol or Aldehyde Oxidation Level Employing 1,1Dimethylallene as the Prenyl Donor. J. Am. Chem. Soc. 2009, 131, 6916-6917. (c) Zbieg, J. R.; Fukuzumi, T.; Krische, M. J. Iridium-Catalyzed Hydrohydroxyalkylation of Butadiene: Carbonyl Crotylation. Adv. Synth. Catal. 2010, 352, 2416-2420. (d) Geary, L. M.; Woo, S. K.; Leung, J. C.; Krische, M. J. Diastereo- and Enantioselective Iridium-Catalyzed Carbonyl Propargylation from the Alcohol or Aldehyde Oxidation Level: 1,3-Enynes as Allenylmetal Equivalents. Angew. Chem., Int. Ed. 2012, 51, 2972-2976. (e) Nguyen, K. D.; Herkommer, D.; Krische, M. J. Enantioselective Formation of All-Carbon Quaternary Centers via C-H Functionalization of Methanol: Iridium-Catalyzed Diene Hydrohydroxymethylation. J. Am. Chem. Soc. 2016, 138, 14210-14213. (f) Holmes, M.; Nguyen, K. D.; Schwartz, L. A.; Luong, T.; Krische, M. J. Enantioselective Formation of $\mathrm{CF}_{3}$-Bearing All-Carbon Quaternary Stereocenters via $\mathrm{C}-\mathrm{H}$ Functionalization of Methanol: Iridium Catalyzed Allene Hydrohydroxymethylation. J. Am. Chem. Soc. 2017, 139, 8114-8117. (g) Xiang, M.; Luo, G.; Wang, Y.; Krische, M. J. Enantioselective IridiumCatalyzed Carbonyl Isoprenylation via Alcohol-Mediated Hydrogen Transfer. Chem. Commun. 2019, 55, 981-984.

(7) For selected examples of Ni-catalyzed reductive coupling of $\pi$-unsaturated pronucleophiles with carbonyl compounds, see: (a) Miller, K. M.; Luanphaisarnnont, T.; Molinaro, C.; Jamison, T. F. Alkene-Directed, Nickel-Catalyzed Alkyne Coupling Reactions. J. Am. Chem. Soc. 2004, 126, 4130-4131. (b) Ng, S.-S.; Jamison, T. F. Highly Enantioselective and Regioselective Nickel-Catalyzed Coupling of Allenes, Aldehydes, and Silanes. J. Am. Chem. Soc. 2005, 127, 7320 7321. (c) Ogoshi, S.; Tonomori, K.; Oka, M.; Kurosawa, H. Reversible Carbon-Carbon Bond Formation between 1,3Dienes and Aldehyde or Ketone on Nickel(0). J. Am. Chem. Soc. 2006, 128, 7077-7086. (d) Kimura, M.; Ezoe, A.; Mori, M.; Iwata, K.; Tamaru, Y. Regio- and Stereoselective NickelCatalyzed Homoallylation of Aldehydes with 1,3-Dienes. J. Am. Chem. Soc. 2006, 128, 8559-8568. (e) Köpfer, A.; Sam, B.; Breit, B.; Krische, M. J. Regiodivergent Reductive Coupling of 2-Substituted Dienes to Formaldehyde Employing Ruthenium or Nickel Catalyst: Hydrohydroxymethylation via Transfer Hydrogenation. Chem. Sci. 2013, 4, 1876-1880.

(8) For an example of Ti-catalyzed allylation of aldehydes with 1,3-dienes, see: Bareille, L.; Le Gendre, P.; Moïse, C. First Catalytic Allyltitanation Reactions. Chem. Commun. 2005, 775-777.

(9) For recent examples of $\mathrm{Cu}$-catalyzed stereoselective addition of $\pi$-unsaturated pronucleophiles to carbonyl derivatives reported by our research group, see: (a) Yang, Y.; Perry, I. B.; Buchwald, S. L. Copper-Catalyzed Enantioselective Addition of Styrene-Derived Nucleophiles to Imines Enabled by Ligand-Controlled Chemoselective Hydrocupration. J. Am. Chem. Soc. 2016, 138, 9787-9790. (b) Yang, Y.; Perry, I. B.; Lu, G.; Liu, P.; Buchwald, S. L. CopperCatalyzed Asymmetric Addition of Olefin-Derived 
Nucleophiles to Ketones. Science 2016, 353, 144-150. (c) Liu, R. Y.; Yang, Y.; Buchwald, S. L. Regiodivergent and Diastereoselective CuH-Catalyzed Allylation of Imines with Terminal Allenes. Angew. Chem., Int. Ed. 2016, 55, 1407714080. (d) Tsai, E. Y.; Liu, R. Y.; Yang, Y.; Buchwald, S. L. A Regio- and Enantioselective $\mathrm{CuH}-\mathrm{Catalyzed} \mathrm{Ketone} \mathrm{Allylation}$ with Terminal Allenes. J. Am. Chem. Soc. 2018, 140, 20072011. (e) Liu, R. Y.; Zhou, Y.; Yang, Y.; Buchwald, S. L. Enantioselective Allylation Using Allene, a Petroleum Cracking Byproduct. J. Am. Chem. Soc. 2019, 141, 2251-2256. (f) Li, C.; Liu, R. Y.; Jesikiewicz, L. T.; Yang, Y.; Liu, P.; Buchwald, S. L. CuH-Catalyzed Enantioselective Ketone Allylation with 1,3Dienes: Scope, Mechanism, and Applications. J. Am. Chem. Soc. 2019, 141, 5062-5070.

(10) For selected reports by other research groups, see: (a) Saxena, A.; Choi, B.; Lam, H. W. Enantioselective CopperCatalyzed Reductive Coupling of Alkenylazaarenes with Ketones. J. Am. Chem. Soc. 2012, 134, 8428-8431. (b) Gui, Y.Y.; Hu, N.; Chen, X.-W.; Liao, L.; Ju, T.; Ye, J.-H.; Zhang, Z.; Li, J.; Yu, D.-G. Highly Regio- and Enantioselective CopperCatalyzed Reductive Hydroxymethylation of Styrenes and 1,3Dienes with $\mathrm{CO}_{2}$. J. Am. Chem. Soc. 2017, 139, 17011-17014. (c) Li, K.; Shao, X.; Tseng, L.; Malcolmson, S. J. 2-Azadienes as Reagents for Preparing Chiral Amines: Synthesis of 1,2Amino Tertiary Alcohols by $\mathrm{Cu}-\mathrm{Catalyzed} \mathrm{Enantioselective}$ Reductive Couplings with Ketones. J. Am. Chem. Soc. 2018, 140, 598-601. (d) Shao, X.; Li, K.; Malcolmson, S. J. Enantioselective Synthesis of Anti-1,2-Diamines by $\mathrm{Cu}-$ Catalyzed Reductive Couplings of Azadienes with Aldimines and Ketimines. J. Am. Chem. Soc. 2018, 140, 7083-7087. (e) Li, M.; Wang, J.; Meng, F. Cu-Catalyzed Enantioselective Reductive Coupling of 1,3-Dienes and Aldimines. Org. Lett. 2018, 20, 7288-7292. (f) Fu, B.; Yuan, X.; Li, Y.; Wang, Y.; Zhang, Q.; Xiong, T.; Zhang, Q. Copper-Catalyzed Asymmetric Reductive Allylation of Ketones with 1,3-Dienes. Org. Lett. 2019, 21, 3576-3580.

(11) (a) Chen, J.-X.; Daeuble, J. F.; Brestensky, D. M.; Stryker, J. M. Highly Chemoselective Catalytic Hydrogenation of Unsaturated Ketones and Aldehydes to Unsaturated Alcohols Using Phosphine-Stabilized Copper(I) Hydride Complexes. Tetrahedron 2000, 56, 2153-2166. (b) Lipshutz, B. H.; Chrisman, W.; Noson, K. Hydrosilylation of Aldehydes and Ketones Catalyzed by $\left[\mathrm{Ph}_{3} \mathrm{P}(\mathrm{CuH})\right]_{6}$. J. Organomet. Chem. 2001, 624, 367-371.

(12) (a) Furuta, K.; Mouri, M.; Yamamoto, H. Chiral (Acyloxy)Borane Catalyzed Asymmetric Allylation of Aldehydes. Synlett 1991, 1991, 561-562. (b) Keck, G. E.; Krishnamurthy, D.; Grier, M. C. Catalytic Asymmetric Allylation Reactions. 3. Extension to Methallylstannane, Comparison of Procedures, and Observation of a Nonlinear Effect. J. Org. Chem. 1993, 58, 6543-6544. (c) Yanagisawa, A.; Nakashima, H.; Ishiba, A.; Yamamoto, H. Catalytic Asymmetric Allylation of Aldehydes Using a Chiral Silver(I) Complex. J. Am. Chem. Soc. 1996, 118, 4723-4724. (d) Ogawa, C.; Sugiura, M.; Kobayashi, S. Stereospecific, Enantioselective Allylation of A-Hydrazono Esters by Using Allyltrichlorosilanes with BINAP Dioxides as Neutral-Coordinate Organocatalysts. Angew. Chem., Int. Ed. 2004, 43, 6491-6493. (e) Kotani, S.; Hashimoto, S.; Nakajima, M. Chiral Phosphine Oxide BINAPO as a Lewis Base Catalyst for Asymmetric Allylation and Aldol Reaction of Trichlorosilyl Compounds. Tetrahedron 2007, 63, 3122-3132. (f) Zhang, Y.; Li, N.; Qu, B.; Ma, S.; Lee, H.; Gonnella, N. C.; Gao, J.; Li, W.; Tan, Z.; Reeves, J. T. et al. Asymmetric Methallylation of Ketones
Catalyzed by a Highly Active Organocatalyst 3,3'-F-BINOL. Org. Lett. 2013, 15, 1710-1713. (g) Silverio, D. L.; Torker, S.; Pilyugina, T.; Vieira, E. M.; Snapper, M. L.; Haeffner, F.; Hoveyda, A. H. Simple Organic Molecules as Catalysts for Enantioselective Synthesis of Amines and Alcohols. Nature 2013, 494, 216.

(13) Colacot, T. J. A Concise Update on the Applications of Chiral Ferrocenyl Phosphines in Homogeneous Catalysis Leading to Organic Synthesis. Chem. Rev. 2003, 103, 31013118 .

(14) Smith, A. B.; Kim, W.-S.; Tong, R. Uniting Anion Relay Chemistry with Pd-Mediated Cross Coupling: Design, Synthesis and Evaluation of Bifunctional Aryl and Vinyl Silane Linchpins. Org. Lett. 2010, 12, 588-591.

(15) In general, away from equilibrium, the Gibbs free energy of reaction $\Delta \mathrm{G}$ differs from the standard free energy of reaction $\Delta \mathrm{G}^{\ddagger}$ by a quantity related to the reaction quotient $Q$ :

$$
\Delta \mathrm{G}-\Delta \mathrm{G}^{*}=R T \ln Q
$$

In our system of interest, the aldehyde concentration is presumably maintained at a very low value, meaning that states involving free aldehyde are lowered in free energy relative to those involving the aldehyde bound to the catalyst. Although this effect is not reflected in Figure 2, which shows only the standard free energies, it is a useful mnemonic to associate the effect of decreasing the steady-state aldehyde concentration with raising the energies of the grey-highlighted states relative to the white ones.

(16) (a) Kagan, H. B.; Dang, T.-P. Asymmetric Catalytic Reduction with Transition Metal Complexes. I. Catalytic System of Rhodium(I) with (-)-2,3-0-Isopropylidene-2,3Dihydroxy-1,4-Bis(Diphenylphosphino)Butane, a New Chiral Diphosphine. J. Am. Chem. Soc. 1972, 94, 6429-6433. (b) Kagan, H. B. In Asymmetric Catalysis; Morrison, J. D., Ed.; Academic Press: New York, 1985; Vol. 5, pp 1-339. (c) Whitesell, J. K. $\mathrm{C}_{2}$-Symmetry and Asymmetric Induction. Chem. Rev. 1989, 89, 1581-1590. (d) Walsh, P.; Kowzlowski, M. Fundamentals of Asymmetric Catalysis; University Science Books: Sausalito, CA, 2008.

(17) For an example of allylation of carbonyl compounds via six-membered Zimmerman-Traxler transition state, see: (a) Grayson, M. N.; Krische, M. J.; Houk, K. N. RutheniumCatalyzed Asymmetric Hydrohydroxyalkylation of Butadiene: The Role of the Formyl Hydrogen Bond in Stereochemical Control. J. Am. Chem. Soc. 2015, 137, 8838-8850. For a review, see: (b) Mejuch, T.; Gilboa, N.; Gayon, E.; Wang, H.; Houk, K. N.; Marek, I. Axial Preferences in Allylation Reactions via the Zimmerman-Traxler Transition State. Acc. Chem. Res. 2013, 46, 1659-1669. 
Table of Contents Graphic:

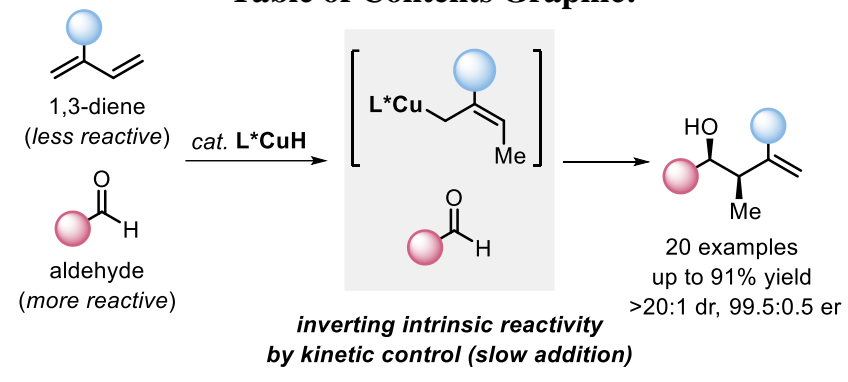

